\title{
ARTICLE
}

\author{
Received 9 Nov 2015 | Accepted 7 Dec 2015 | Published 20 Jan 2016 DOl: 10.1038/ncomms10431
}

\section{ssODN-mediated knock-in with CRISPR-Cas for large genomic regions in zygotes}

Kazuto Yoshimi ${ }^{1,2}$, Yayoi Kunihiro ${ }^{1,3}$, Takehito Kaneko ${ }^{1}$, Hitoshi Nagahora ${ }^{4}$, Birger Voigt $^{1}$ \& Tomoji Mashimo ${ }^{1,3}$

The CRISPR-Cas system is a powerful tool for generating genetically modified animals; however, targeted knock-in (KI) via homologous recombination remains difficult in zygotes. Here we show efficient gene $\mathrm{KI}$ in rats by combining CRISPR-Cas with single-stranded oligodeoxynucleotides (ssODNs). First, a 1-kb ssODN co-injected with guide RNA (gRNA) and Cas 9 messenger RNA produce GFP-KI at the rat Thy 1 locus. Then, two gRNAs with two 80-bp ssODNs direct efficient integration of a 5.5-kb CAG-GFP vector into the Rosa26 locus via ssODN-mediated end joining. This protocol also achieves $\mathrm{KI}$ of a 200-kb BAC containing the human SIRPA locus, concomitantly knocking out the rat Sirpa gene. Finally, three gRNAs and two ssODNs replace 58-kb of the rat Cyp2d cluster with a 6.2-kb human CYP2D6 gene. These ssODN-mediated $\mathrm{KI}$ protocols can be applied to any target site with any donor vector without the need to construct homology arms, thus simplifying genome engineering in living organisms.

\footnotetext{
${ }^{1}$ Institute of Laboratory Animals, Graduate School of Medicine, Kyoto University, Kyoto 606-8501, Japan. ${ }^{2}$ Mouse Genomics Resource Laboratory, National Institute of Genetics, Shizuoka 411-8540, Japan. ${ }^{3}$ Institute of Experimental Animal Sciences, Graduate School of Medicine, Osaka University, Osaka 565-0871, Japan. ${ }^{4}$ BioDynamics Laboratory Inc., Tokyo 113-0033, Japan. Correspondence and requests for materials should be addressed to T.M. (e-mail: mashimo@iexas.med.osaka-u.ac.jp).
} 
G enetically modified (GM) animals play an important role in the study of gene functions and for understanding the mechanisms of human diseases. Zinc finger nucleases, transcription activator-like effector nucleases (TALENs) and clustered regularly interspaced short palindromic repeats (CRISPR)-associated (Cas) nucleases are efficient genome engineering tools for generating GM animals via microinjection into fertilized eggs ${ }^{1-6}$. The injected nucleases recognize long stretches of DNA sequence and introduce DNA double-strand breaks (DSBs), which are generally repaired via non-homologous end-joining (NHEJ), a process that introduces small insertions or deletions (indels) at the repair junction. This process allows efficient generation of animals with knockout (KO) alleles at targeted sequences ${ }^{7-9}$. Targeted knock-in (KI) can be also engineered via homologous recombination (HR) by co-injection of donor plasmids, including large DNA fragments such as green fluorescent protein (GFP) cassettes, with any of the above mentioned nucleases ${ }^{10-15}$. This was traditionally achieved by constructing targeting vectors with two homology arms of $0.5-1 \mathrm{~kb}$ on either side of the insert DNA. However, HR-mediated $\mathrm{KI}$ is less efficient than NHEJ-mediated $\mathrm{KO}$, because the canonical HR pathway for DSB repair in mammalian cells or embryos is less effective than the NHEJ pathway ${ }^{16-18}$.

Recently, single-stranded oligodeoxynucleotides (ssODNs) have been used as donor templates in combination with the engineered nucleases for efficient targeted insertion of small DNA fragments. ssODN-mediated $\mathrm{KI}$ in mammalian cells occurs via homology-directed repair (HDR) and is more efficient than using double-stranded donor plasmids ${ }^{18-21}$. In mice, targeted KI with ssODN donors and engineered nucleases has been reported to be highly efficient ${ }^{22-25}$. We have also shown the efficient generation of ssODN-mediated $\mathrm{KI}$ in rats by recovering three recessive coat-colour phenotypes ${ }^{26}$.

In this study, ssODNs in combination with methodical CRISPR-Cas fine tuning are employed to KI and replace large genomic regions of the rat genome with human genes. We first append polyadenine tails (poly(A)) to plasmids expressing Cas9 mRNA, to increase the efficacy of genome editing with the CRISPR-CasCRISPR-Cas system. Using Cas9-poly(A), we employ two approaches to conduct targeted KI with relatively long DNA fragments, such as the GFP sequence. The first use long ssODNs (lsODNs) as a targeting donor, which are newly synthesized by a method using nicking endonucleases. The second involve co-injection of two guide RNAs (gRNAs) to act as 'scissors', to cut target sites in genomic DNA and the donor plasmid DNA, and two short ssODNs to act as 'paste', to ligate the ends of the cut sites. This method enable efficient KI of plasmid DNAs including a bacterial artificial chromosome (BAC) of $200 \mathrm{~kb}$. The significant technical advantage of this ssODN-mediated end joining approach is its simplicity, as there is no need to construct homology arms in the donor vector, which is especially difficult to perform in larger BACs.

\section{Results}

Poly-A tail elongation enhances genome editing efficiency. Poly(A) tails play an important role in the stability and the translation of most eukaryotic mRNAs ${ }^{27-29}$. To increase the translation efficiency of the Cas9 mRNA in zygotes, we appended an 81-bp poly(A) signal to the transcription terminator of the Cas9-2A-GFP plasmid (Addgene ID\#44719) and injected in vitro-transcribed Cas9 mRNA $\left(100 \mathrm{ng}_{\mu} \mathrm{l}^{-1}\right)$ into rat male pronuclei of fertilized eggs (Fig. 1a). Three hours after injection, eggs treated with Cas9-GFP-poly(A) $\quad(n=23)$ showed significantly higher fluorescence intensity compared with eggs injected with Cas9-GFP without poly(A) $(n=20)$ (Fig. 1b,c). The higher expression in the Cas9-GFP-poly(A)-injected eggs persisted at the two-cell stage $24 \mathrm{~h}$ after injection (Fig. 1b,c). In addition, eggs injected with Cas9-GFP-poly(A) did not show any significant abnormal features or delayed development compared with eggs without poly(A) during the 24 -h culture period. These findings indicate that an elongated poly(A) tail can enhance the translation of Cas 9 mRNA in rat embryos.

Next, we co-injected $50 \mathrm{ng}^{-1} \mathrm{l}^{-1}$ gRNA and Cas9-GFP-poly(A) mRNA targeting the rat Tyrosinase (Tyr) gene ${ }^{26}$ into fertilized rat eggs (Supplementary Table 1). After $24 \mathrm{~h}$, two-cell embryos were collected and genomic DNA was amplified to analyse NHEJ-mediated KO mutations at the targeted Tyr locus (Fig. 1d). Cas9-poly(A)-injected embryos showed a higher rate of NHEJ-mediated indel mutations $(7 / 19,36.8 \%)$ compared with wild-type Cas9-injected embryos $(2 / 20,10.0 \%)$ (Fisher's exact test: $P<0.05$; Supplementary Table 1 ).

We then co-injected $50 \mathrm{ng}^{-1}$ ssODNs with gRNA and Cas9-poly(A) mRNA (without GFP) into fertilized rat eggs, to recover the albino phenotype (a missense mutation in Tyr) by ssODN-mediated single nucleotide polymorphism exchange, as previously described ${ }^{26}$. Transfer of the injected embryos into pseudopregnant female rats resulted in the birth of 31 pups, among which 20 (64.5\%) showed NHEJ-mediated KO mutations and $13(41.9 \%)$ showed HDR-mediated KI mutations, indicated by phenotype recovery from albino to black hooded (Fig. 1e, Table 1 and Supplementary Fig. 1). Among the 13 animals with a KI mutation, 7 also showed NHEJ-mediated indels. These mutation rates for KO and KI by Cas9-poly(A) are significantly higher than the $23.1 \% \mathrm{KO}$ and $7.7 \% \mathrm{KI}$ found in our previous study without poly(A) enhancement ${ }^{26}$ (Fisher's exact test: $P<0.005$ for KO and $P<0.05$ for KI). Neither insertions nor deletions were observed in the $13 \mathrm{KI}$ rats at any off-target sites across the whole genome, examined according to standard criteria $^{26}$ (Supplementary Table 2).

GFP KI at the rat Thy1 locus using long ssODNs. We have previously used the CRISPR-Cas system together with a 119-bp ssODN, to accomplish targeted integration of a 19-bp nucleotide fragment at the agouti signalling protein locus (Asip) to recover the non-agouti phenotype ${ }^{26}$. To integrate longer DNA fragments, such as a GFP reporter cassette, we synthesized an 837-bp lsODN consisting of GFP-coding sequences and two 60-bp homology arms at either end of the GFP sequence (Fig. 2a and Supplementary Fig. 2). We then designed gRNA targeting the start codon (ATG) of the rat thymus cell antigen 1 locus (Thy1) to produce THY1-GFP fusion protein. Microinjection of gRNA:Thy1-ATG, Cas9-poly(A) mRNA, together with $50 \mathrm{ng}_{\mu 1} \mathrm{l}^{-1}$ lsODN into Wistar rat zygotes resulted in the birth of 48 pups (Fig. 2b, Supplementary Fig. 3 and Supplementary Table 3). PCR and subsequent sequence analysis revealed that 37 pups (77.1\%) showed NHEJ-mediated KO mutations and 5 pups (13.5\%) carried HDR-mediated KI sequences at the targeted site (Fig. 2c and Supplementary Fig. 4). However, all KI alleles included partial insertion or deletion at the $5^{\prime}$-region of the Thy1 gene, which prevented translation of the complete GFP-THY1 fusion protein (Fig. 2d).

As the observed deletion mutations on the $5^{\prime}$-side of the integrated lsODN sequences were probably caused by the $5^{\prime}-3^{\prime}$ exonuclease activity of the DSB repair system ${ }^{18}$ (see Discussion), we targeted the stop codon (TGA) of the rat Thyl gene with a complementary lsODN $(-)$, which included the $2 \mathrm{~A}$ peptide, GFP and $3^{\prime}$-untranslated region sequences, as well as an extended $5^{\prime}$-homology arm of $300 \mathrm{bp}$, to avoid the effect of possible exonuclease activity (Fig. 2a and Supplementary Fig. 5). We obtained 36 pups, of which 33 (91.7\%) showed KO mutations 
a

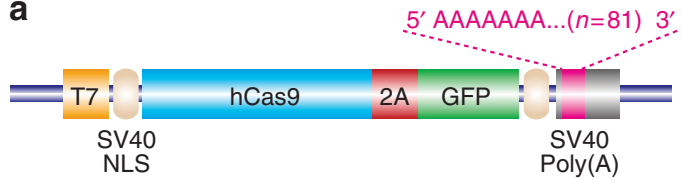

b
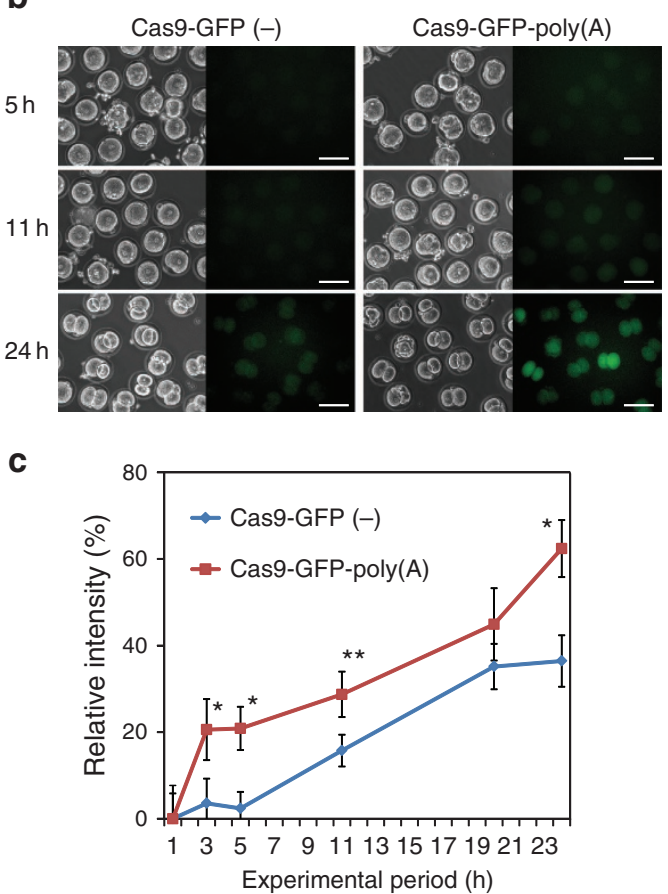

d

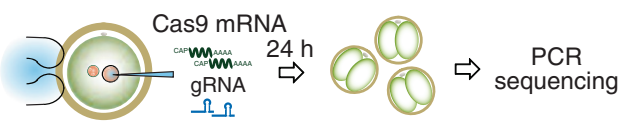

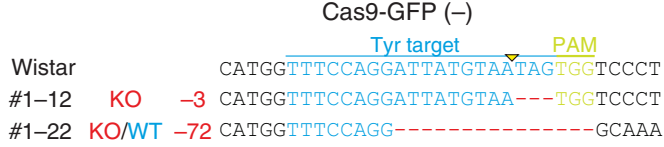

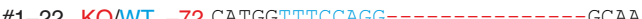

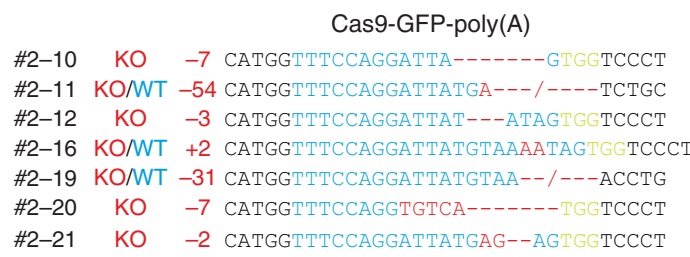

e

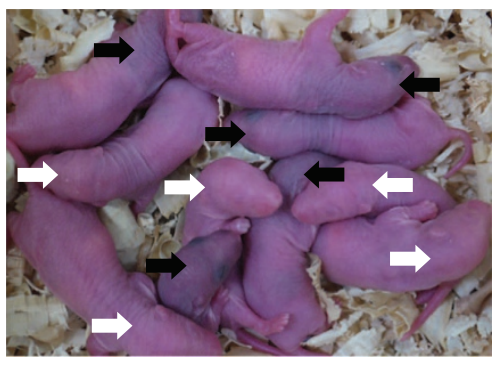

$41.9 \%(13 / 31 \mathrm{KI}$ pups/total offspring)

Figure 1 | Poly(A) tail elongation enhances genome-editing efficiency in zygotes. (a) Schematic representation of the Cas9-2A-GFP plasmid. An 81-bp poly (A) was added to the $3^{\prime}$-untranslated region (UTR) of the construct. (b) In vitro-transcribed Cas9 mRNA (100 ng $\left.\mu\right|^{-1}$ ) was injected into pronuclei of rat fertilized eggs. Fluorescence microscopy images were captured after injection. (c) The fluorescence intensity of oocytes was estimated as relative intensity with the highest intensity of oocytes after 24 -h incubation set at $100 \%$. ${ }^{\star} P<0.05$ and ${ }^{\star \star} P<0.001$ by Student's $t$-test. Error bars represents.e.m.

(d) Co-injection of gRNA (50 ng $\mathrm{I}^{-1}$ ) targeting the rat tyrosinase (Tyr) gene with Cas9 mRNA into rat fertilized eggs resulted in NHEJ-mediated KO mutations at the targeted sites (Supplementary Fig. 1). Two-cell embryos were collected and genomic DNA was amplified by PCR to detect NHEJ-mediated $\mathrm{KO}$ mutations at the targeted Tyr locus. (e) Co-injection of ssODN (50 ng $\mu \mathrm{l}^{-1}$ ) with gRNA and Cas9-poly(A) mRNA into rat fertilized eggs corrected the albino phenotype (white arrows) by HDR-mediated single nucleotide polymorphism (SNP) exchange, to produce the black-hooded phenotype (black arrows).

(Supplementary Fig. 6) and 7 showed GFP-positive alleles (Fig. 2e). Among these 7 pups, two (numbers 11 and 18) were positive for a PCR product amplified across 1 of the Thy1-GFP junction sequences and 4 pups (11.1\%, numbers 7, 9, 10 and 24) were positive for PCR products amplified across both of the junction sequences. Sequence analysis revealed that all four pups carried entire sequences for rat Thy1, the $2 \mathrm{~A}$ peptide and GFP (Fig. 2f). Finally, in these pups, GFP fluorescence was observed in brain sections of cortical neurons and cerebellar Purkinje cells (Fig. 2g).

CAG-GFP plasmid KI at the Rosa26 locus. In recent times, we have successfully deleted a 7-kb fragment of endogenous retroviral DNA from the genome of coat-colour-modified rats ${ }^{26}$. The experiment was designed with two gRNAs to cut on either side of the retroviral insertion and one ssODN that included homology arms to link the two DSBs of the original rat DNA. Here we applied a similar design to knock in longer plasmids, including the $1.2-\mathrm{kb}$ CAG promoter and a 0.8 -kb GFP cassette sequence (Supplementary Fig. 7). Following the previously reported NHEJ-dependent KI strategy ${ }^{30}$, we constructed two gRNAs: one targeting the rat Rosa26 locus to cleave genomic DNA (Supplementary Fig. 8) and the other targeting $5^{\prime}$ of the
CAG promoter sequence for concurrent cleavage of the plasmid DNA. We also designed two 80-bp ssODNs to ligate the two cut ends, as shown in Fig. 3a. We named this strategy 'two-hit by gRNA and two oligos with a targeting plasmid' (2H2OP). We co-injected a mix of $100 \mathrm{ng}^{-1}$ of the Cas9-poly(A) mRNA,

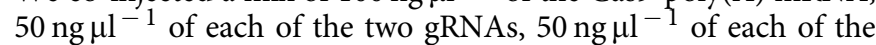
two ssODNs and $5 \mathrm{ng}^{-1}$ of the CAG-GFP plasmid into Wistar rat embryos (Table 1). Of the 17 pups delivered, 4 expressed GFP in the whole body (Fig. $3 \mathrm{~b}$ and Supplementary Fig. 9). PCR analysis showed specific amplification of GFP sequences in 4 of the 17 pups (numbers 6, 7, 8 and 11) (Fig. 3c). Primer sets designed for either side of each Rosa26 and CAG-GFP junction (F1-R3 or F3-R1 in Fig. 3a) amplified the junction sequences in pups numbers 6,7 and 11 . However, a primer set designed to amplify across the cleavage site of the CAG-GFP plasmid produced a PCR product in pup number 8 (Fig. 3c). Sequence analysis of the 4 pups revealed that number 11 had accurate conjunction at both of the 2 cut ends, number 6 had a 6-bp deletion on one side and number 7 had a several-base-pair deletion and insertion on both sides (Fig. 3d).

Crossing rat numbers 8 and 11 with Wistar rats resulted in the faithful transmission of the GFP allele to the next generation (Supplementary Table 4). Again, no off-target effect was observed at any candidate sites in these rats (Supplementary Table 2). 


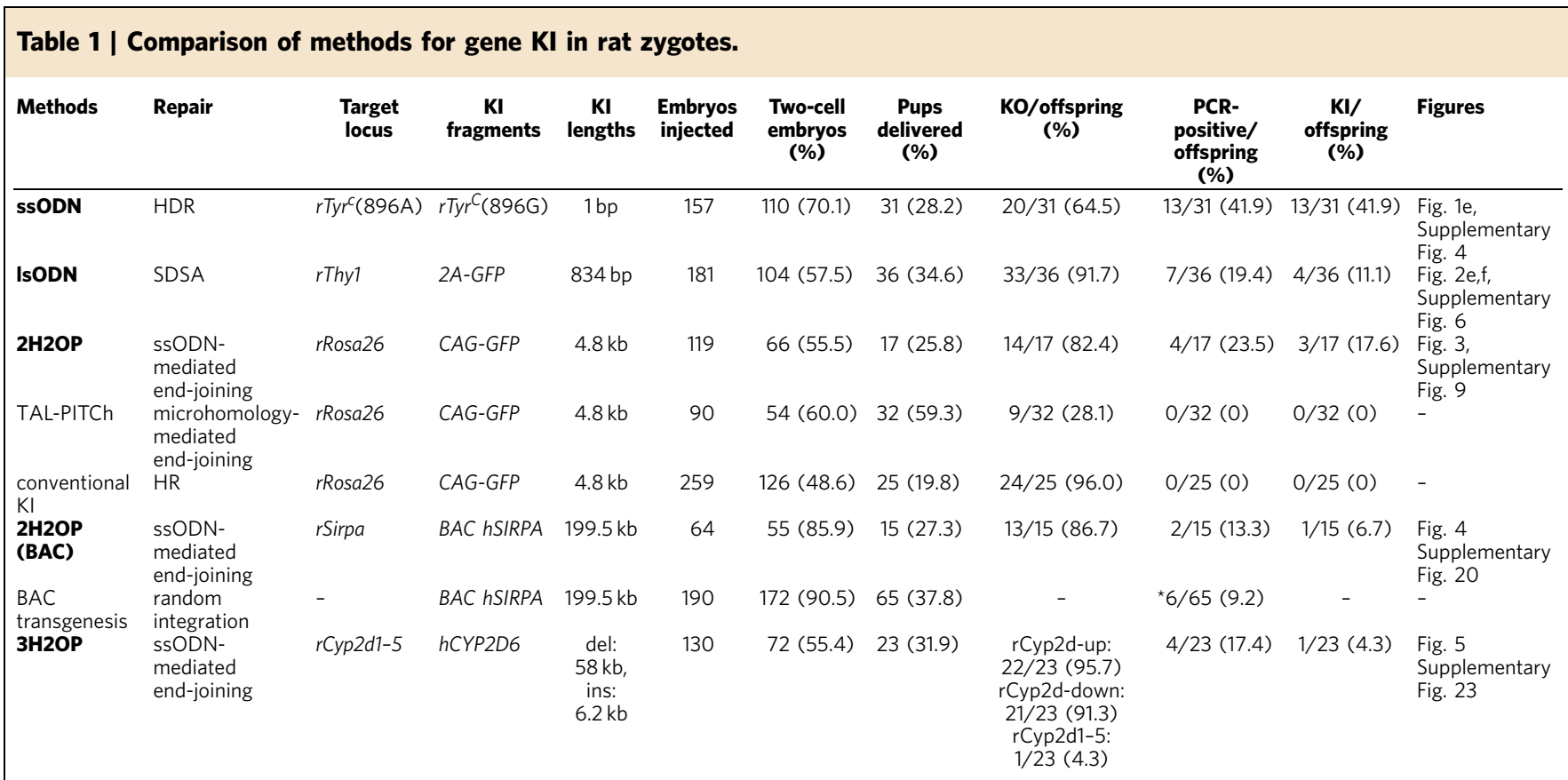

2H2OP, two-hit by gRNA and two oligos with a targeting plasmid; BAC, bacterial artificial chromosome; GFP, green fluorescent protein; gRNA, guide RNA; HDR, homology-directed repair; HR, homologous recombination; KI, knock-in; IsODN, long ssODN; SDSA, synthesis-dependent strand annealing; SNP, single nucleotide polymorphism; ssODN, single-stranded oligodeoxynucleotide; TALPITCh, TALEN-mediated precise integration into target chromosome system.

Cas9-poly(A) mRNA, gRNA(s), ssODN(s) and/or a donor plasmid were injected into fertilized rat eggs. ssODN-mediated SNP exchange was implemented as previously described 20 . Tyr $\mathrm{c}$, an albino mutation allele (896A): Tyr $\mathrm{C}$, a wild-type allele (896G). IsODN consisting of GFP sequences flanking a 60-bp and a 300-bp homology arms was synthesized by a method using nicking endonucleases (see Methods). The donor plasmid comprising CAG-GFP sequences flanking a 515-bp and a 645-bp homology arms was used in conventional KI. In TAL-PITCh method, which is microhomologymediated end-joining-dependent KI methods reported by Nakade et al. ${ }^{15}$, a pair of TALENs, which recognizes the same target site at the rat Rosa26 locus and on the TAL-PITCh vector, were co-injected with the TAL-PITCh vector containing CAG-GFP sequences. In BAC transgenesis, linierized BAC clone (RP11-993C19) was injected into fertilized rat eggs. ${ }^{*}$ Among 65 delivered pups, 6 were positive for transgene of the human SIRPA.

Southern blot analysis confirmed that rat number 11 had a singlecopy GFP KI allele at the Rosa26 locus, and that rat number 8 had an allele of three GFP transgene copies inserted at a random chromosomal site (Supplementary Fig. 10). Fluorescent in situ hybridization (FISH) analysis using labelled CAG-GFP probes in homozygous offspring from rat number 11 (W-Rosa26 $6^{\mathrm{em} 1}$ $(C A G-G F P) K y o$, which are deposited in the National BioResource Project for the rat (NBRP-Rat): No. 0770) confirmed that the KI allele was integrated into rat chromosome 4q41-q42 where Rosa26 is located (Supplementary Fig. 11). Heterozygous W-Rosa26 $6^{\text {em1(- }}$ $C A G-G F P) K y o$ rats showed intense GFP fluorescence from only one copy of the KI allele (Supplementary Fig. 12). They also showed uniform expression in most cells and tissues examined, such as the brain, liver, heart and pancreas (Supplementary Fig. 13).

Considering the repair system for CRISPR-mediated DSBs with ssODNs, where $5^{\prime}-3^{\prime}$ exonuclease activity may cause indel mutations, we again used a complementary $\operatorname{ssODN}(-)$ upstream of the Rosa26 cut site (Supplementary Fig. 14a). We observed that the complementary $\operatorname{ssODN}(-)$ increased the KI efficiency slightly but not significantly $(28.6 \%$; 6/21; Supplementary Table 5), although 5 of 6 KI rats still showed several base-pair deletions or insertions on either side of the KI (Supplementary Fig. 14b). This $2 \mathrm{H} 2 \mathrm{OP}$ approach was further applied to C57BL/6J mice, to knock in CAG-GFP at the mouse Rosa26 locus (Supplementary Fig. 15). Of the 31 pups that were delivered, all $31(100 \%)$ showed indel mutations at the mouse Rosa26 locus (Supplementary Fig. 16). Sequence analysis revealed that three pups $(9.7 \%)$ expressing GFP in the whole body carried the KI allele with conjunct sequences between Rosa26 and the CAG-GFP plasmid (Supplementary Fig. 17).

Human BAC KI at the rat Sirpa locus. To investigate whether much larger plasmids, such as BAC clones, could be knocked in to a targeted genomic region, we used the $2 \mathrm{H} 2 \mathrm{OP}$ approach to knock out the rat Sirpa gene and concomitantly knock in the human SIRPA gene, which is contained within the 200-kb BAC clone, RP11-933C19 (Fig. 4a). Cas9 mRNA, two gRNAs targeting exon 2 of rat Sirpa (Supplementary Fig. 18) and the PI-SceI site of pBACe3.6 (Supplementary Fig. 19), and two ssODNs for ligation of the rat genome sequences and the 933C19 BAC sequence, were injected into Wistar rat embryos. We obtained 15 pups from 64 embryos injected (Table 1). PCR analysis revealed that 13 pups $(86.7 \%)$ carried KO alleles at the rat Sirpa locus (Supplementary Fig. 20), and that 2 pups (13.3\%) were positive for the human SIRPA gene (Fig. 4b). Primer sets designed to amplify across each side of the rat Sirpa locus and PI-SceI junction (F1-R3 or F3-R1 in Fig. 4a) amplified the conjunct sequences in rat number 2 (Fig. 4b). Sequence analysis revealed that rat number 2 had accurate conjunction at both of the two cut ends (Fig. 4c).

To examine whether the complete $200-\mathrm{kb}$ sequence or only a part of it was inserted at the targeted site, we performed PCR analysis using primer sets amplifying the whole human SIRPA gene and BAC vector sequences, all of which were positive for KI rat number 2 (Supplementary Fig. 21). FISH analysis of rat KI number 2 using labelled BAC vector probes revealed that the KI allele was integrated into rat chromosome 7q41-q42 where Sirpa is located (Fig. 4d). Finally, reverse transcriptase-PCR using primer sets amplifying hSIRPA or rSirpa complementary DNA showed unique expression of the human SIRPA gene in the KI rats (Fig. 4e).

Genomic humanization of the Cyp2d cluster genes. Although human cytochrome P450 2D6 (CYP2D6) is one of the most important enzymes involved in the metabolism of xenobiotics, in particular clinically used drugs, the orthologous rat gene remains unknown ${ }^{31,32}$. However, the gene encoding CYP2D6 is assumed 
a 1-hit 1-oligo (1H1O) by long ssODN (ISODN)

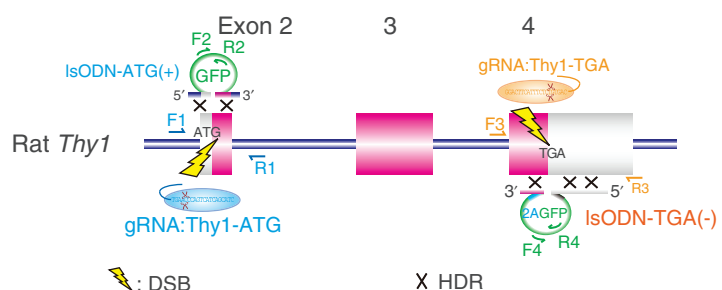

C

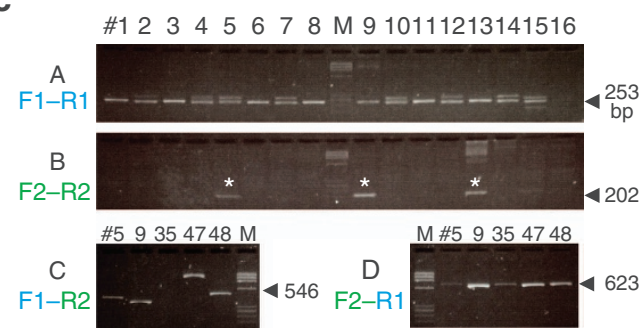

d

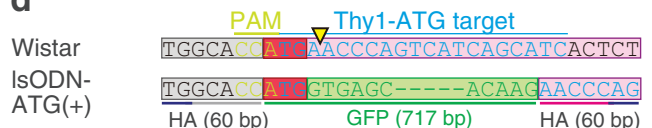

\#5 KI/KO/WTTTGG------- (148bp) -E(566bp) AAGAACCC

\#9 KI/WT CTT -------(170bp) -E(713bp) AAGAACCC

\#35 KI/WT TGGCA A (192bp)-E(522bp) AAGAACCC

\#47 KI TGGCA AAA+515bp

\#48 KI/WT TGGCA A - (88bp) -

$13.5 \%$ (5/48 KI pups/total offspring)

e

f b
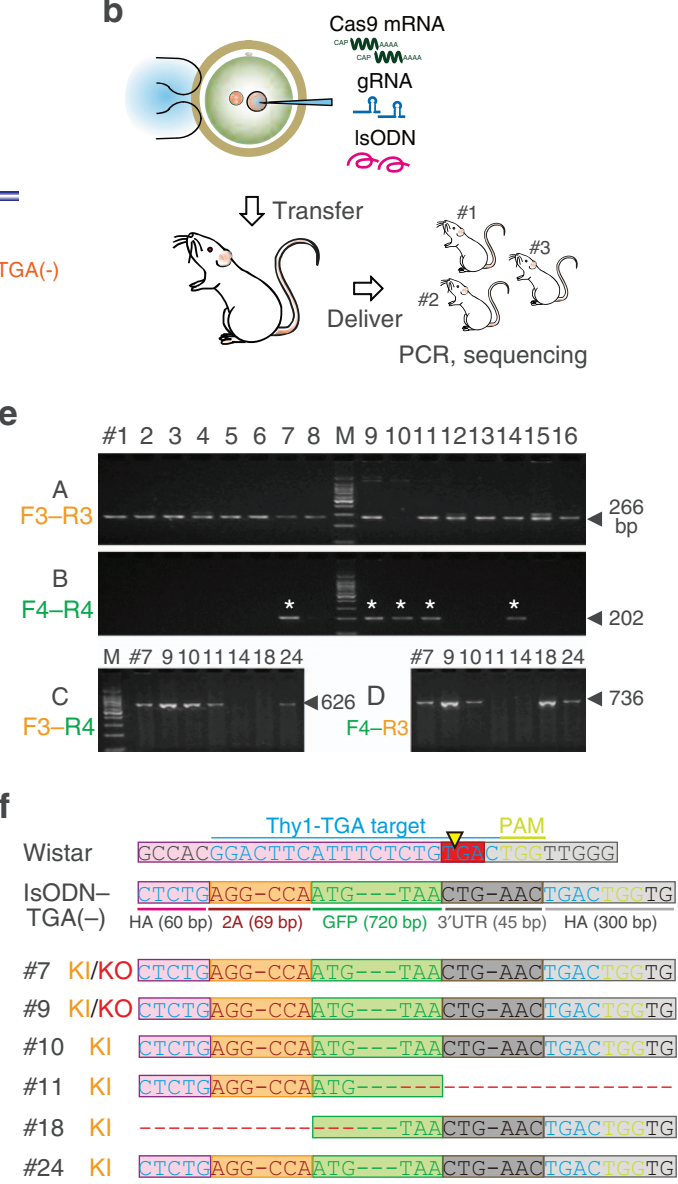

$11.1 \%$ (4/36 KI pups/total offspring)

g

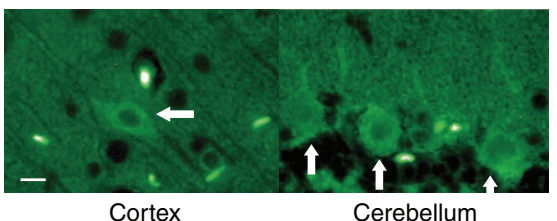

Figure 2 | IsODNs with CRISPR-Cas mediates KI at the rat Thy1 locus. (a) Schematic representation of the rat thymus cell antigen 1 (Thy1) gene, gRNA targeting sites (Thy1-ATG and Thy1-TGA), primer sets (F1-4 and R1-4) and the IsODNs, each containing a GFP cassette and two 60-300 bp homology sequences. (b) Schematic of gRNA, Cas9 mRNA and IsODN co-injection into rat fertilized eggs to generate founders carrying the KI mutation. (c) PCR analysis of pups (1-16) derived from co-injection of gRNA:Thy1-ATG, Cas9 mRNA and IsODN-ATG( + ) with primer sets, F1,2 and R1,2, as indicated in a (see also Supplementary Table 3). Many pups show indel mutations at the targeted Thy1-ATG locus (also shown in Supplementary Fig. 4).

(A) The product size of the wild-type Wistar allele (arrowed) is 253-bp. Some pups showed a GFP-positive PCR band (asterisk). (B) PCR-positive band with primer sets, F1 and R2 (C) or F2 and R1 (D) indicate ligation at either side of the Thy1-ATG and the GFP. Various indel mutations were found in C, but not in $\mathrm{d}$, after comparison with the predicted PCR size indicated by arrows. (d) Sequence analysis of the GFP-positive pups showed HDR-mediated KI alleles with partial deletions of 5'-GFP coding sequences. (e) Co-injection of gRNA:Thy1-TGA, Cas9 mRNA and the complementary IsODN( - ) with extended 300-bp homology arms into rat fertilized eggs. Many pups showed indel mutations at the targeted Thy1-TGA locus (Supplementary Fig. 6). (A) The product size of the wild-type Wistar allele (arrowed) is $266 \mathrm{bp}$. Some pups showed a GFP-positive PCR band (asterisk). (B) PCR analysis of the pups with primer sets F3 and R4 (C) or F4 and R3 (D) showed the ligation at either side of Thy1-TGA and GFP. (f) Sequence analysis of 7 GFP-positive pups showed that four rats (numbers 7, 9, 10 and 24) carried complete sequences of HDR-mediated KI alleles, including rat Thy1, 2A peptide and GFP. The remaining three failed to be sequenced at the conjunction sites. (g) KI rat number 24 showed GFP-positive neurons in the cortex and GFP-positive Purkinje cells in the cerebellum (arrows). Blood vessels were also GFP positive. Scale bar, $10 \mu \mathrm{m}$.

to be located in the rat Cyp2d1-5 gene cluster. To delete the whole $58-\mathrm{kb}$ region containing five orthologous rat genes, Cyp2d1-5, and to knock in a 6.2-kb sequence of the human CYP2D6 gene, we designed three gRNAs: one targeting upstream of the rat Cyp2d2 locus, the second downstream of the rat Cyp2d4 locus (Supplementary Fig. 22) and the third targeting the plasmid that contains human CYP2D6 (Fig. 5a). We then co-injected two 120-bp ssODNs together with the CYP2D6 plasmid, to ligate each cut end of the rat Cyp2d1-5 cluster region with the cut ends of the human CYP2D6 plasmid. We obtained 23 pups delivered from 72 transferred embryos (Table 1 and Supplementary Fig. 23). PCR analysis with primer sets for the human CYP2D6 locus and the rat Cyp2d loci indicated that rat numbers $1,3,8$ and 18 carried the human CYP2D6 gene, and that rat number 2 carried the deletion 
a

2-hit 2-oligo with plasmids (2H2OP)

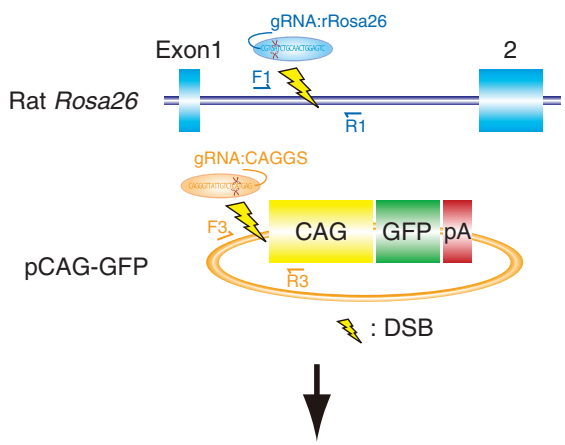

Rat Rosa26 ${ }^{\text {em(CAG-GFP })}$

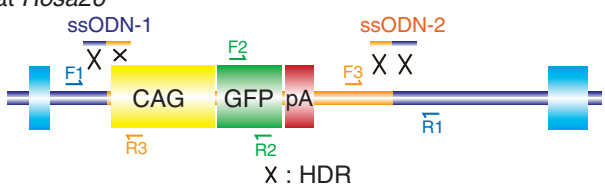

C

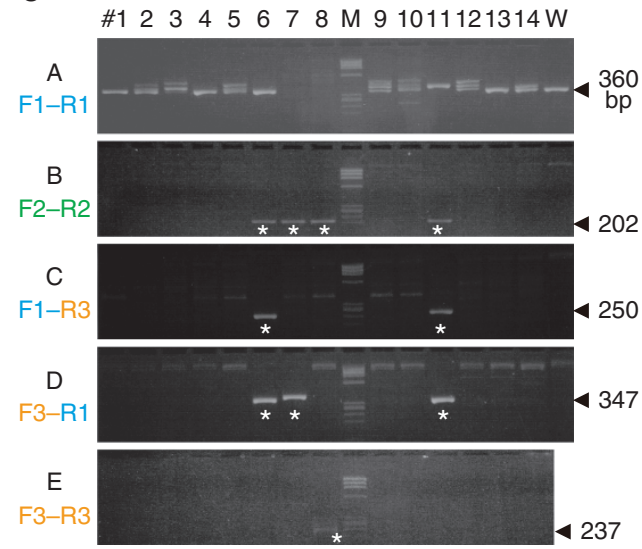

b

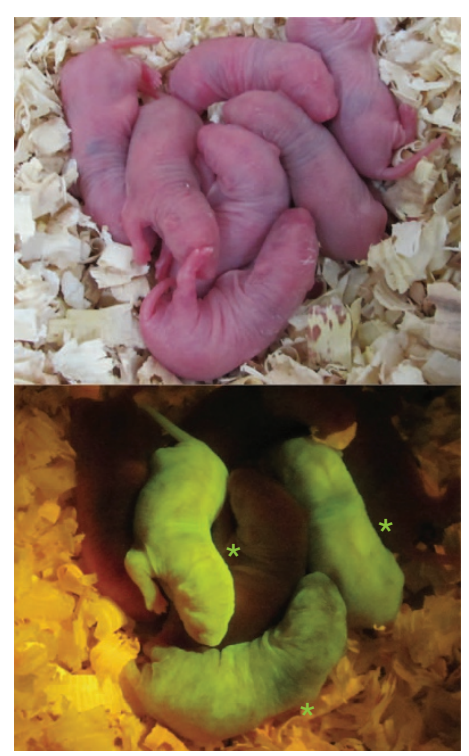

d

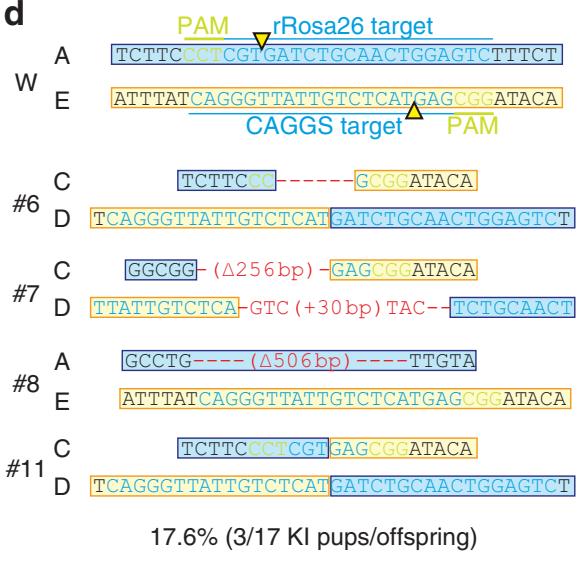

Figure 3 | CAG-GFP KI rats generated using the CRISPR-Cas system. (a) Schematic representation of the two-hit two-oligo with plasmid (2H2OP) method. In the first step, Cas9, together with two gRNAs targeting the rat Rosa26 locus and the CAG promoter in the GFP plasmids, cut the target sites. In the second step, two ssODNs ligate each cut end to join the genomic DNA and the plasmid DNA via HDR. (b) Photos of pups delivered from Wistar rat embryos injected with a mix of Cas9-poly(A) mRNA, two gRNAs, two ssODNs and CAG-GFP plasmids (upper). Some of the pups (numbers 6, 7 and 8) expressed GFP at a high level in the body (asterisk) (lower). (c) PCR analysis of the pups with primer sets F1-3 and R1-3, as indicated in a. Many pups showed indel mutations at the targeted Rosa26 locus, compared with the 360-bp wild-type allele indicated by arrows (Supplementary Fig. 9) (A). Four pups showed a GFP-positive PCR band (asterisk) (B). Three pups, numbers 6, 7 and 11, were positive for the ligation between either side of Rosa26 and CAG-GFP $(C, D)$. Rat number 8 was positive for the uncut CAG sequences (E). Arrows indicate the predicted PCR size. (d) Sequence analysis of pups indicated that rat number 11 carried accurate conjunction at both cut ends.

allele of the whole rat Cyp2d cluster (primer sets $\mathrm{C}$ and set D, respectively in Fig. 5b). Primer sets $\mathrm{E}$ and F, designed to amplify either end of the of the human CYP2D6 insertion into the rat Cyp2d cluster, amplified the conjunct sequences in rat number 18, indicating that gene(s) replacement of the rat Cyp2d cluster with the human CYP2D6 gene was accomplished by the 3-hit 2-oligo with plasmids $(3 \mathrm{H} 2 \mathrm{OP})$ method (Fig. 5b). The replacement was confirmed by sequence analysis as shown in Fig. $5 \mathrm{c}$. FISH analysis of the number $18 \mathrm{KI}$ rat using labelled plasmid probes revealed that the human CYP2D6 allele was integrated into rat chromosome 7q34 where the rat Cyp2d is located (Supplementary Fig. 24).

\section{Discussion}

Plasmids consisting of homology arms, the targeting cassette and antibiotic selection markers are generally used for targeted KI via
HR in mammalian cells, including embryonic stem and induced pluripotent stem cells. The recent combination of this approach with site-specific endonucleases, such as CRISPR-Cas, drastically increased targeting efficiency in these cultured cells ${ }^{33-35}$. In zygotes, targeted KI with plasmids via classical HR has also been reported by several groups ${ }^{13-15,24}$. However, the efficiency is still not ideal, because selection markers are not available. In this study, we have shown two new approaches that use the CRISPR-Cas system. The first used a synthetic long ssODN containing a GFP cassette and successfully knocked in GFP at the $3^{\prime}$-end of the rat Thy 1 gene. The second approach, $2 \mathrm{H} 2 \mathrm{OP}$, used two short ssODNs for the integration of a longer plasmid, which allowed the efficient integration of a CAG-GFP vector into the rat Rosa26 locus. In addition, $2 \mathrm{H} 2 \mathrm{OP}$ allowed KI of a 200-kb BAC containing the human SIRPA gene at the rat Sirpa locus and also achieved replacement of the rat Cyp2d cluster genes with the 
a

2-hit 2-oligo with BAC plasmids (2H2OP)
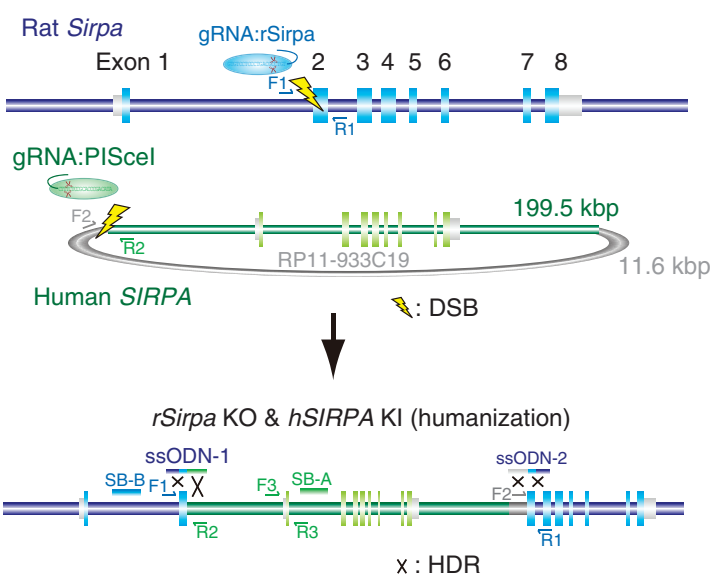

d

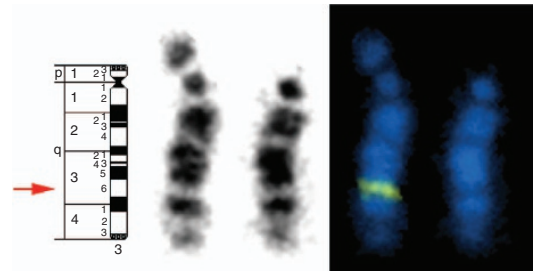

b

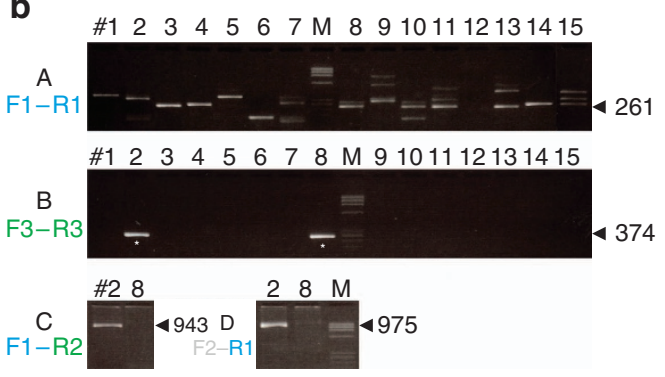

C

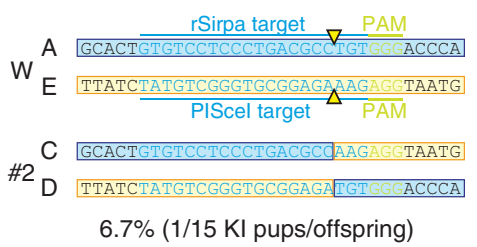

e

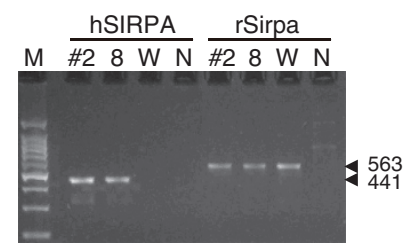

Figure 4 | Human BAC KI at the SIRPA locus. (a) Schematic representation of the BAC KI by 2H2OP. The rat Sirpa gene was knocked out by KI of the 200kb BAC (RP11-933C19), which contains the human SIRPA locus. (b) PCR analysis of pups with primer sets F1-3 and R1-3, as indicated in a. Many pups showed indel mutations at the targeted rat Sirpa locus compared with the 261-bp wild-type allele (arrow) (A) and two pups showed a human SIRPA-positive PCR band (asterisk) (B). Rat number 2 was positive for ligation between both sides of rat Sirpa and human SIRPA (C,D). Arrows indicated the predicted PCR fragment sizes. (c) Sequence analysis of pups indicated that rat number 2 carried accurate conjunction at both cut ends. (d) The heterozygous SIRPA-KI allele was confirmed by FISH on rat chromosome 3q35 (arrow). (e) Reverse transcriptase-PCR analysis of human SIRPA mRNA in the liver of rat numbers 2 and $8 . \mathrm{N}$, negative control; W, control Wistar.

human CYP2D6 gene. To the best of our knowledge, this is the first report of the targeted KI of such a long BAC fragment and the replacement of a large genomic region containing a cluster of genes by a single injection of unmodified donor DNA, ssODNs and CRISPR-Cas into rodent embryos.

Recent studies showed the high efficiency of CRISPRCas-mediated KI in zebrafish by concurrent cleavage of donor plasmid DNA and genomic DNA using an NHEJ-dependent DNA repair system ${ }^{36,37}$. More recently, microhomologymediated end-joining-dependent targeted KI of donor plasmid DNA by TALEN or CRISPR-Cas was reported as TALENmediated precise integration into target chromosome system (PITCh) or CRISPR-mediated PITCh methods respectively, in human cells and frogs ${ }^{38}$. These KI technologies are both achieved by the concurrent cleavage of genomic DNA and donor plasmids without using ssODN, but have not been used in mouse and rat zygotes. As shown in Table 1 , we injected Platinum TALENs ${ }^{39}$ into rat zygotes targeting the Rosa26 locus with a TALENmediated-PITCh vector containing CAG-GFP sequences, resulting in several TALEN-mediated KO pups (9/32), but no pups with GFP KI alleles (0/32) at the Rosa26 locus. The apparent difference in KI efficiency between fish-frogs and rodents might reflect the different dependence on the DNA repair system between species. In contrast, the $2 \mathrm{H} 2 \mathrm{OP}$ method in this study provided several pups with GFP KI alleles (3/17) at the same Rosa26 locus, suggesting that co-injected ssODNs can support the ligation of CRISPR-Cas-mediated DSBs of the template into genomic target DNA. The increased ligation efficiency via sSODN-mediated end joining was also observed in our previous studies $^{26}$.
Several groups have reported HR-dependent KI in rats using targeting vectors and zinc finger nuclease ${ }^{10}$, TALEN $^{13,40}$ and CRISPR $^{14,15}$. The efficiency of HR-mediated KI is strongly dependent on the gene being targeted, but technical conditions are also a factor. Ma et al. ${ }^{14}$ reported $>50 \% \mathrm{KI}$ efficiency (6/11 pups) at the rat $C c k$ locus; however, most other studies have reported a lower efficiency $(1-30 \%)$ or failure of $\mathrm{KI}$ at certain genes. In our experiments, co-injection of Cas9-polyA, gRNA targeting Rosa26 and a donor plasmid comprising CAG-GFP sequences flanking 515 and 645-bp homology arms into rat zygotes resulted in no pups with KI alleles (0/25; Table 1). Homology arms are usually cloned into targeting vectors for HRbased experiments, whereas no homology arms are required in existing plasmids in $2 \mathrm{H} 2 \mathrm{OP}$. The notable advantage of $2 \mathrm{H} 2 \mathrm{OP}$ is the targeted KI of much larger DNA fragments, such as BAC clones, or the replacement of large gene clusters, illustrated in Figs 4 and 5, respectively, without the need to modify the donor vectors. However, the overall efficiency of this BAC KI or cluster gene replacement was low, at $6.7 \%$ and $4.3 \%$, respectively. Our injection of the same BAC clone into rat zygotes to produce conventional transgenic animals resulted in a $9.2 \%$ efficiency (6/65 pups), where the BAC was randomly integrated (Table 1). Such a low efficiency of BAC transgenesis has been reported by other groups $(1-4 \%)^{41,42}$. The efficiency for the integration or deletion of large regions by $2 \mathrm{H} 2 \mathrm{OP}$ may depend on the size of the targeted region as previously reported ${ }^{43,44}$.

The disadvantage of $2 \mathrm{H} 2 \mathrm{OP}$ is the high rate of indel mutations at ssODN-mediated conjunction sites (Fig. 3d). The lsODN approach could circumvent this problem for precise $\mathrm{KI}$. In the experiment using lsODN targeting the start codon of the Thy1 
a

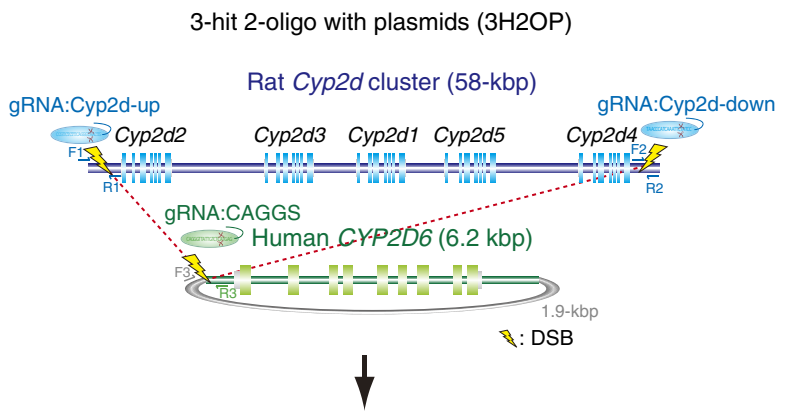

Replacement of Cyp2d genes (humanization)

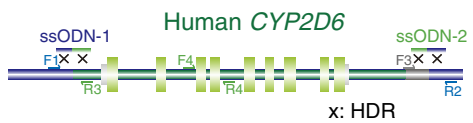

b

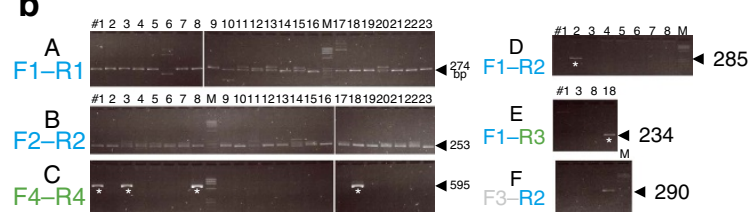

C

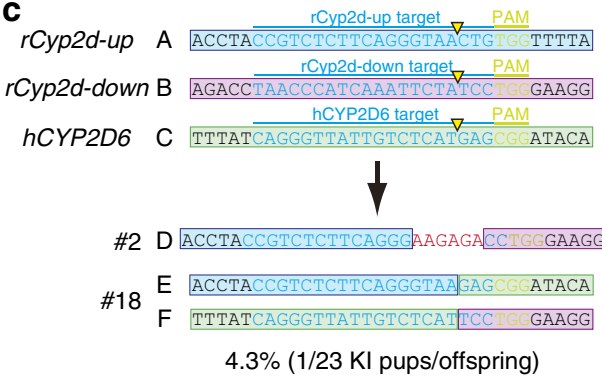

Figure 5 | Replacement of the rat Cyp2d cluster with the human CYP2D6 gene. (a) Schematic representation of the 3H2OP method to replace the rat Cyp2d cluster genes, Cyp2d1-5 (58 kb), with the orthologous human CYP2D6 gene (6.2 kb). Three gRNAs for upstream and downstream of the rat Cyp2d cluster and for the human CYP2D6 plasmid cut the targeting sites, and two ssODNs ligate to each cut end. (b) PCR analysis of pups with primer sets F1-4 and R1-4 as indicated in a. Many pups showed indel mutations compared with the wild-type alleles upstream (274 bp) and downstream (253 bp) of Cyp2d, indicated by arrows ( $A$ and B, respectively). Four pups showed a positive PCR band for the human CYP2D6 gene (asterisk) (C). Rat number 18 was positive for ligation between both sides of the rat Cyp2d cluster and human CYP2D6 (D,E). Rat number 2 showed a large deletion from upstream to downstream of the rat Cyp2d cluster (E). Arrows indicate the predicted PCR fragment size. (c) Sequence analysis of pups indicated that rat number 18 carried the accurate conjunction at both cut ends.

locus, many deletion mutations were observed on the $5^{\prime}$-side of the integrated lsODN sequences (Fig. 2d), probably because of the $5^{\prime}-3^{\prime}$ exonuclease activity of the DSB repair system. These observations provide some clues about the underlying mechanism for repairing CRISPR-mediated DSBs with single-stranded ODNs. We hypothesize that this repair system is the synthesisdependent strand annealing pathway, which is one of the major HR pathways for repairing DSBs ${ }^{45,46}$ (Supplementary Fig. 25). In the synthesis-dependent strand annealing pathway, after resection of the $5^{\prime}$-strand of the DSB, the $3^{\prime}$-strand of the ssODN anneals to the homologous sequences on the $3^{\prime}$-strand of the DSB. Then, DNA synthesis occurs from the $3^{\prime}$-end of the DSB, to copy the remaining $5^{\prime}$-strand of the ssODN. A second annealing occurs with the other $3^{\prime}$-end of the DSB after removal of the ssODN. Finally, gap filling and ligation complete the repair event ${ }^{18,19,45,46}$. The $5^{\prime}$-degradation of ssODNs by exonuclease activity or the removal of the ssODN by helicase activity might concurrently occur with DNA synthesis, which causes incomplete repair, such as indel mutations (Supplementary Fig. 25). In this process, microhomology sequences between the $3^{\prime}$-strand of the DSB and the newly synthesized $3^{\prime}$-strand of the ssODN may also cause indel mutations. In this study, extension of the $5^{\prime}$-side of the homology arms up to 300-bp drastically ameliorated the deletion mutations on the $5^{\prime}$-side of the lsODN sequences, providing the precise 2A-GFP KI at the end of the Thy1 locus (Fig. 2f,g). The limitation of the lsODN approach is the maximal length of the synthetic lsODN, which is currently limited to $\sim 3 \mathrm{~kb}$ (Nagahora et al., unpublished data).

In this study, we also demonstrated that poly(A) tail elongation enhances the translation efficiency of Cas9 mRNA in zygotes, thereby increasing the efficiency of CRISPR-mediated genome editing (Fig. 1). Poly(A) tails generally play an important role in the stability and translation of most eukaryotic mRNAs ${ }^{27,28,29}$. In vertebrate oocytes, maternal mRNAs are mostly dormant with relatively short poly(A) tails, usually fewer than 20 nucleotides $27,28,29$. In contrast, the length of the poly(A) tail is strongly associated with translational efficiency ${ }^{47}$. Interestingly, this association is observed only in oocytes or early-stage embryos, but not in somatic cells or tissues, which suggests that a translational control switch exists in early embryos ${ }^{47}$. It is also reported that attaching synthetic tails or increasing the length of an mRNA increases its translation in Xenopus ${ }^{48}$, Drosophila ${ }^{49}$ and mouse oocytes ${ }^{50,51}$. Consistent with previously published papers $^{51}$, our study confirms that increased levels of Cas9 in embryos produced neither severe toxicity nor off-target effects in other chromosomal regions.

In conclusion, we have established new ssODN-mediated KI approaches with the CRISPR-Cas system, which are applicable to any target site, any species and with any donor vector without the need of attaching homology arms. These technologies facilitate easy and flexible genome engineering in living organisms, such as the production of rainbow animals with colourful reporter genes or cross-species GM (for example, humanized) animals.

\section{Methods}

Animals. F344/Stm (NBRP-Rat No. 0140) rats were provided by the National Bio Resource Project for the Rat in Japan (www.anim.med.kyoto-u.ac.jp/nbr). Jcl:Wistar rats and C57BL/6JJcl mice were obtained from CLEA Japan Inc. (Tokyo, Japan). The animals were kept under conditions of $50 \%$ humidity and a $14: 10 \mathrm{~h}$ light:dark cycle. The newly developed W-Rosa ${ }^{e m 1(C A G-G F P) K y o}$ rats (NBRP-Rat No. 0770) were deposited into the National Bio Resource Project-Rat in Japan. They were fed a standard pellet diet (F-2, Oriental Yeast Co., Tokyo, Japan) and tap water ad libitum. Animal care and experiments conformed to the Guidelines for Animal Experiments of Kyoto University and were approved by the Animal Research Committee of Kyoto University.

Preparation of Cas9 and gRNA vectors and donor DNA. Plasmid vectors expressing hCas9 (ID\#41815) were obtained from the Addgene repository (www.addgene.org/CRISPR) and were modified by addition of the T7 promoter and the SV40 nuclear localization signal at the amino terminus of hCas9, using an In-Fusion HD cloning kit (Takara Bio, Shiga, Japan) according to the manufacturer's protocol. An 81-bp poly(A) was also added to the $3^{\prime}$-untranslated region of hCas9 using the In-Fusion cloning kit. To measure expression intensity in rat embryos, the 2A peptide and an Enhanced GFP cassette (from pCas9-2A-GFP: Addgene ID\#44719) was inserted downstream of hCas9. Cas9 mRNA was transcribed in vitro using a mMESSAGE mMACHINE T7 Ultra Kit (Life Technologies, Carlsbad, CA, USA) from linearized plasmids and was purified using a MEGAClear kit (Life Technologies).

To design the gRNAs, software tools (crispr.genome-engineering.org) predicting unique target sites throughout the rat genome were used. gRNAs 
were transcribed in vitro using a MEGAshortscript T7 Transcription Kit (Life Technologies) from synthetic double-stranded DNAs that included a T7 promoter, 20-bp target sequences and gRNA tail sequences, obtained from Integrated DNA Technologies (IA, USA). To prepare gRNA, oligonucleotides designed for target sites were also cloned into BsaI-digested pDR274 vectors (Addgene ID\#42250). The target sites and sequences of the ssODNs in the rat genome are shown in Supplementary Table 6 .

ssODNs were obtained from Integrated DNA Technologies. pCAGGS was obtained from RIKEN BRC, which is participating in the National Bio-Resource Project of the Ministry of Education, Culture, Sports, Science and Technology, Japan. DNA encoding enhanced GFP was inserted into pCAGGS and was verified by sequence analysis. A BAC clone containing the human SIRPA gene (RP11-993C19) was obtained from Life Technologies. BAC DNA was purified using NucleoBond BAC 100 (Takara Bio) according to the manufacturer's protocol.

Long ssODNs. lsODNs were prepared using an LsODN Preparation Kit (Biodynamics Laboratory Inc., Tokyo, Japan) according to the manufacturer's protocol. A DNA fragment comprising a GFP cassette and homology arms was cloned between the BspQI and the BsrDI sites of pLSODN-1. The resulting plasmid was digested with nicking endonucleases Nt.BspQI and Nb.BsrDI. The nicked plasmid was subject to denaturing agarose gel electrophoresis containing urea. BynaMarker Prestain Marker for RNA High conc (Biodynamics Laboratory Inc.) was used as a visible molecular weight marker to monitor electrophoresis in real time. After electrophoresis, the gel was stained with crystal violet solution, which visualized three bands, a single-strand DNA fragment comprising GFP cassette and homology arms, a liner single-strand vector DNA and a single-strand circular whole-plasmid DNA. The band corresponding to a single-strand DNA fragment comprising GFP cassette and homology arms was excised and extracted according to the manufacturer's protocol. LsODNs were aliquoted and stored at $-80^{\circ} \mathrm{C}$, to avoid degradation by repetitive freeze-thaw.

Microinjections into rat and mouse embryos. Rat and mouse females were superovulated by injection with gonadotropin serum from pregnant mares (PMSG: Aska Pharmaceutical Co., Tokyo, Japan) and human chorionic gonadotropin (Aska Pharmaceutical Co.). Next, pronuclear-stage embryos were collected from superovulated rat or mouse females. They were cultured in a modified KrebsRinger bicarbonate medium or KSOM medium (ARK Resource, Kumamoto, Japan) before and after microinjections. Using a micromanipulator (Narishige,

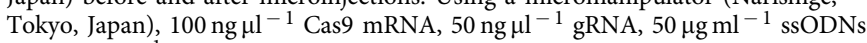
and $5 \mu \mathrm{g} \mathrm{ml}^{-1}$ plasmids were microinjected into the male pronuclei of embryos. Injected embryos were selected randomly for each experiment. They were cultured in modified Krebs-Ringer bicarbonate or KSOM medium overnight and divided two-cell embryos were transferred into pseudopregnant females.

Gene expression of $\mathbf{K I}$ and mutation detection assay. The expression level of GFP in embryos was measured using a BIOREVO immunofluorescence microscope (Keyence, Osaka, Japan). After incubation for $24 \mathrm{~h}$, two-cell embryos were collected and genomic DNA was amplified using the GenomePlex Single Cell Whole Genome Amplification Kit (Sigma Aldrich, St Louis, MO, USA). After purification of the DNA, CRISPR-Cas-mediated mutations at target sites were analysed by direct sequencing.

For mutation detection, genomic DNA was extracted from tail biopsies using a GENEXTRACTOR TA-100 automatic DNA purification system (Takara Bio). The PCR products amplified with specific primer sets (Supplementary Table 6) were directly sequenced using the BigDye terminator v3.1 cycle sequencing mix and the standard protocol for an Applied Biosystems 3130 DNA Sequencer (Life Technologies).

Total RNA was extracted using Isogen reagent (Nippon Gene) from the spleen of 5-week-old rats. First strand cDNA was synthesized from $5 \mu \mathrm{g}$ of total RNA that had been treated with DNase using an oligo(dT)12-18 primer and SuperscriptII reverse transcriptase (Invitrogen). PCR was performed with the primers for human SIRPA and rat Sirpa described in Supplementary Table 6.

Off-target analysis. Potential off-target sites in the rat genome (rn5) were identified using the latest version of the CRISPR Design Tool website (crispr.mit.edu). All potential sites were ranked by the off-target hit score based on the predicted specificity. High-ranked potential sites were sequenced in the founders to identify any off-target sites (Supplementary Table 2).

Southern blotting. Genomic DNA was purified from the liver of all founders using phenol-chloroform extraction. After digestion of the DNA with appropriate restriction enzymes, $10-\mu \mathrm{g}$ samples of digested DNA were separated by electrophoresis on $0.8 \%$ agarose gels and then blotted onto positively charged nylon membranes (Roche, Basel, Switzerland). Ultraviolet cross-linked membranes were hybridized with ${ }^{32} \mathrm{P}$-labelled DNA probes (synthesized by random primed labelling), washed and exposed to X-ray film.
Fluorescent in situ hybridization. Spleen cells were cultured in $10 \mathrm{ml}$ of RPMI1640 medium (Sigma) supplemented with fetal bovine serum (20\%), concanavalin A $\left(3 \mu \mathrm{g} \mathrm{ml}^{-1}\right)$ and lipopolysaccharide $\left(10 \mu \mathrm{g} \mathrm{ml}^{-1}\right)$ for $68 \mathrm{~h}$. Before harvesting, 5-bromo- $2^{\prime}$-deoxyuridine (Sigma) was added for $3.5 \mathrm{~h}$ at a concentration of $30 \mu \mathrm{g} \mathrm{ml}^{-1}$ and then colcemid (Sigma) was added for $30 \mathrm{~min}$ at a concentration of $0.02 \mu \mathrm{g} \mathrm{ml}^{-1}$. The culture was then treated with hypotonic solution $(75 \mathrm{mM} \mathrm{KCl})$ for $20 \mathrm{~min}$ and washed three times in fixative (3:1 methanol:acetic acid). After drying overnight, the metaphase spreads were stained with Hoechst 33258 and ultraviolet irradiated for $4 \mathrm{~min}$ at $70^{\circ} \mathrm{C}$, to visualize chromosome banding patterns.

The plasmids containing the transgene were labelled by nick translation with digoxigenin-11-dUTP (Roche). The labelled probes were mixed with sonicated salmon sperm DNA and rat cot-1 DNA in hybridization solution. The probe was applied to metaphase spreads, denatured at $70^{\circ} \mathrm{C}$ for $5 \mathrm{~min}$ and hybridized overnight at $37^{\circ} \mathrm{C}$. The hybridized slide was washed and probe signals were detected with anti-digoxigenin-Cy3 antibody. After mounting with anti-fade mounting solution, FISH images were captured with the CW4000 FISH application programme (Leica Microsystems Imaging Solution, Cambridge, UK) using a cooled charge-coupled device camera mounted on a Leica DMRA2 microscope.

\section{References}

1. Mashimo, T. Gene targeting technologies in rats: zinc finger nucleases, transcription activator-like effector nucleases, and clustered regularly interspaced short palindromic repeats. Dev. Growth Differ. 56, 46-52 (2014).

2. Kim, H. \& Kim, J. S. A guide to genome engineering with programmable nucleases. Nat. Rev. Genet. 15, 321-334 (2014).

3. Hsu, P. D., Lander, E. S. \& Zhang, F. Development and applications of CRISPR-Cas9 for genome engineering. Cell 157, 1262-1278 (2014).

4. Carroll, D. Genome engineering with targetable nucleases. Annu. Rev. Biochem. 83, 409-439 (2014).

5. Sander, J. D. \& Joung, J. K. CRISPR-Cas systems for editing, regulating and targeting genomes. Nat. Biotechnol. 32, 347-355 (2014).

6. Mali, P., Esvelt, K. M. \& Church, G. M. Cas9 as a versatile tool for engineering biology. Nat. Methods 10, 957-963 (2013).

7. Li, D. et al. Heritable gene targeting in the mouse and rat using a CRISPR-Cas system. Nat. Biotechnol. 31, 681-683 (2013).

8. Li, W., Teng, F., Li, T. \& Zhou, Q. Simultaneous generation and germline transmission of multiple gene mutations in rat using CRISPR-Cas systems. Nat. Biotechnol. 31, 684-686 (2013).

9. $\mathrm{Hu}, \mathrm{X}$. et al. Heritable gene-targeting with gRNA/Cas9 in rats. Cell Res. $\mathbf{2 3}$, 1322-1325 (2013).

10. Cui, X. et al. Targeted integration in rat and mouse embryos with zinc-finger nucleases. Nat. Biotechnol. 29, 64-67 (2011).

11. Meyer, M., de Angelis, M. H., Wurst, W. \& Kuhn, R. Gene targeting by homologous recombination in mouse zygotes mediated by zinc-finger nucleases. Proc. Natl Acad. Sci. USA 107, 15022-15026 (2010).

12. Sommer, D. et al. Efficient genome engineering by targeted homologous recombination in mouse embryos using transcription activator-like effector nucleases. Nat. Commun. 5, 3045 (2014).

13. Remy, S. et al. Efficient gene targeting by homology-directed repair in rat zygotes using TALE nucleases. Genome Res. 24, 1371-1383 (2014).

14. Ma, Y. et al. Generation of eGFP and Cre knockin rats by CRISPR/Cas9. FEBS J. 281, 3779-3790 (2014).

15. Shao, Y. et al. CRISPR/Cas-mediated genome editing in the rat via direct injection of one-cell embryos. Nat. Protoc. 9, 2493-2512 (2014).

16. Sonoda, E., Hochegger, H., Saberi, A., Taniguchi, Y. \& Takeda, S. Differential usage of non-homologous end-joining and homologous recombination in double strand break repair. DNA Repair (Amst) 5, 1021-1029 (2006).

17. Shrivastav, M., De Haro, L. P. \& Nickoloff, J. A. Regulation of DNA double-strand break repair pathway choice. Cell Res. 18, 134-147 (2008).

18. Peng, Y. et al. Making designer mutants in model organisms. Development 141, 4042-4054 (2014).

19. Storici, F., Snipe, J. R., Chan, G. K., Gordenin, D. A. \& Resnick, M. A. Conservative repair of a chromosomal double-strand break by single-strand DNA through two steps of annealing. Mol. Cell. Biol. 26, 7645-7657 (2006).

20. Radecke, S., Radecke, F., Cathomen, T. \& Schwarz, K. Zinc-finger nucleaseinduced gene repair with oligodeoxynucleotides: wanted and unwanted target locus modifications. Mol. Ther. 18, 743-753 (2010).

21. Chen, F. et al. High-frequency genome editing using ssDNA oligonucleotides with zinc-finger nucleases. Nat. Methods 8, 753-755 (2011)

22. Wefers, B. et al. Direct production of mouse disease models by embryo microinjection of TALENs and oligodeoxynucleotides. Proc. Natl Acad. Sci. USA 110, 3782-3787 (2013).

23. Wang, H. et al. One-step generation of mice carrying mutations in multiple genes by CRISPR/Cas-mediated genome engineering. Cell 153, 910-918 (2013).

24. Yang, H. et al. One-step generation of mice carrying reporter and conditional alleles by CRISPR/Cas-mediated genome engineering. Cell 154, 1370-1379 (2013). 
25. Wu, Y. et al. Correction of a genetic disease in mouse via use of CRISPR-Cas9. Cell Stem Cell 13, 659-662 (2013).

26. Yoshimi, K., Kaneko, T., Voigt, B. \& Mashimo, T. Allele-specific genome editing and correction of disease-associated phenotypes in rats using the CRISPR-Cas platform. Nat. Commun. 5, 4240 (2014).

27. Richter, J. D. Cytoplasmic polyadenylation in development and beyond. Microbiol. Mol. Biol. Rev. 63, 446-456 (1999).

28. Goldstrohm, A. C. \& Wickens, M. Multifunctional deadenylase complexes diversify mRNA control. Nat. Rev. Mol. Cell Biol. 9, 337-344 (2008).

29. Weill, L., Belloc, E., Bava, F. A. \& Mendez, R. Translational control by changes in poly(A) tail length: recycling mRNAs. Nat. Struct. Mol. Biol. 19, 577-585 (2012).

30. Maresca, M., Lin, V. G., Guo, N. \& Yang, Y. Obligate ligation-gated recombination (ObLiGaRe): custom-designed nuclease-mediated targeted integration through nonhomologous end joining. Genome Res. 23, 539-546 (2013).

31. Scheer, N. et al. Modeling human cytochrome P450 2D6 metabolism and drug-drug interaction by a novel panel of knockout and humanized mouse lines. Mol. Pharmacol. 81, 63-72 (2012).

32. Wang, X., Li, J., Dong, G. \& Yue, J. The endogenous substrates of brain CYP2D. Eur. J. Pharmacol. 724, 211-218 (2014).

33. Cong, L. et al. Multiplex genome engineering using CRISPR/Cas systems. Science 339, 819-823 (2013).

34. Mali, P. et al. RNA-guided human genome engineering via Cas9. Science 339, 823-826 (2013)

35. Cho, S. W., Kim, S., Kim, J. M. \& Kim, J. S. Targeted genome engineering in human cells with the Cas9 RNA-guided endonuclease. Nat. Biotechnol. 31, 230-232 (2013).

36. Auer, T. O., Duroure, K., Concordet, J. P. \& Del Bene, F. CRISPR/Cas9mediated conversion of eGFP- into Gal4-transgenic lines in zebrafish. Nat. Protoc. 9, 2823-2840 (2014).

37. Auer, T. O., Duroure, K., De Cian, A., Concordet, J. P. \& Del Bene, F. Highly efficient CRISPR/Cas9-mediated knock-in in zebrafish by homologyindependent DNA repair. Genome Res. 24, 142-153 (2014).

38. Nakade, S. et al. Microhomology-mediated end-joining-dependent integration of donor DNA in cells and animals using TALENs and CRISPR/Cas9. Nat. Commun. 5, 5560 (2014).

39. Sakuma, T. et al. Repeating pattern of non-RVD variations in DNA-binding modules enhances TALEN activity. Sci. Rep. 3, 3379 (2013).

40. Ponce de Leon, V., Merillat, A. M., Tesson, L., Anegon, I. \& Hummler, E. Generation of TALEN-mediated GRdim knock-in rats by homologous recombination. PLOS ONE 9, e88146 (2014).

41. Ma, B. et al. Human antibody expression in transgenic rats: comparison of chimeric IgH loci with human $\mathrm{VH}, \mathrm{D}$ and $\mathrm{JH}$ but bearing different rat C-gene regions. J. Immunol. Methods 400-401, 78-86 (2013).

42. Takahashi, R. et al. Generation of transgenic rats with YACs and BACs: preparation procedures and integrity of microinjected DNA. Exp. Anim. 49, 229-233 (2000)

43. Lee, H. J., Kim, E. \& Kim, J. S. Targeted chromosomal deletions in human cells using zinc finger nucleases. Genome Res. 20, 81-89 (2010).

44. Canver, M. C. et al. Characterization of genomic deletion efficiency mediated by clustered regularly interspaced palindromic repeats (CRISPR)/Cas9 nuclease system in mammalian cells. J. Biol. Chem. 289, 21312-21324 (2014).

45. Sung, P. \& Klein, H. Mechanism of homologous recombination: mediators and helicases take on regulatory functions. Nat. Rev. Mol. Cell Biol. 7, 739-750 (2006).
46. Liu, C. et al. A fine-scale dissection of the DNA double-strand break repair machinery and its implications for breast cancer therapy. Nucleic Acids Res. 42, 6106-6127 (2014).

47. Subtelny, A. O., Eichhorn, S. W., Chen, G. R., Sive, H. \& Bartel, D. P. Poly(A)-tail profiling reveals an embryonic switch in translational control. Nature 508, 66-71 (2014).

48. Barkoff, A., Ballantyne, S. \& Wickens, M. Meiotic maturation in Xenopus requires polyadenylation of multiple mRNAs. EMBO J. 17, 3168-3175 (1998).

49. Salles, F. J., Lieberfarb, M. E., Wreden, C., Gergen, J. P. \& Strickland, S. Coordinate initiation of Drosophila development by regulated polyadenylation of maternal messenger RNAs. Science 266, 1996-1999 (1994).

50. Yamagata, K. et al. Noninvasive visualization of molecular events in the mammalian zygote. Genesis 43, 71-79 (2005).

51. Fujii, W., Kawasaki, K., Sugiura, K. \& Naito, K. Efficient generation of large-scale genome-modified mice using gRNA and CAS9 endonuclease. Nucleic Acids Res. 41, e187 (2013).

\section{Acknowledgements}

This study was supported in part by Grants-in-Aid for Scientific Research from the Japan Society for the Promotion of Science (26290033 to T.M. and 25890011 to K.Y.). We thank Machiko Hayashi, Kosuke Hattori, Yoshiki Miyasaka and Takuji Yoshimura for experimental assistance.

\section{Author contributions}

T.M. designed the study, analysed the data and wrote the paper. K.Y. and Y.K. performed the animal experiments, PCR and sequence analyses. K.Y., T.K. and B.V. performed microinjection of CRISPR-Cas into mouse and rat embryos. H.N. synthesized the lsODNs used in this study. All authors have read and approved the manuscript before submission.

\section{Additional information}

Supplementary Information accompanies this paper at http://www.nature.com/ naturecommunications

Competing financial interests: H.N. is an employee of BioDynamics Laboratory Inc. All other authors declare no competing financial interest.

Reprints and permission information is available online at http://npg.nature.com/ reprintsandpermissions/

How to cite this article: Yoshimi, K. et al. ssODN-mediated knock-in with CRISPR-Cas for large genomic regions in zygotes. Nat. Commun. 7:10431 doi: 10.1038/ncomms10431 (2016).

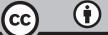

This work is licensed under a Creative Commons Attribution 4.0 International License. The images or other third party material in this article are included in the article's Creative Commons license, unless indicated otherwise in the credit line; if the material is not included under the Creative Commons license, users will need to obtain permission from the license holder to reproduce the material. To view a copy of this license, visit http://creativecommons.org/licenses/by/4.0/ 